\section{References}

1. Yasui H, Kado H, Nakano E, Yonenaga K, Mitani A, Tomita Y, et al. Primary repair of interrupted aortic arch and severe aortic stenosis in neonates. J Thorac Cardiovasc Surg. 1987;93:539-45.

2. Rowlatt UF, Rimoldi HJA, Lev M. The quantitative anatomy of the normal child's heart. Pediatr Clin North Am. 1963;10:499-588.

3. McCrindle BW, Tchervenkov CI, Konstantinov IE, Williams WG, Neirotti RA, Jacobs ML, et al. Risk factors associated with mortality and interventions in 472 neonates with interrupted aortic arch: a Congenital Heart Surgeons Society study. J Thorac Cardiovasc Surg. 2005; 129:343-50

4. Ohye RG, Kagisaki K, Lee LA, Mosca RS, Goldberg CS, Bove EL. Biventricular repair for aortic atresia or hypoplasia and ventricular septal defect. J Thorac Cardiovasc Surg. 1999;118:648-53.

5. Black MD, Smallhorn JF, Freedom RM. Aortic atresia with a ventricular septal defect: modified single-stage neonatal biventricular repair. Ann Thorac Surg. 1999;67:751-5.

\title{
A new cannula for innominate artery cannulation
}

\author{
Paolo Stassano, MD, Antonino Musumeci, MD, Gabriele lannelli, MD, Giuseppe D'Alise, CCCP, and \\ Michele Mottola, MD, Naples, Italy
}

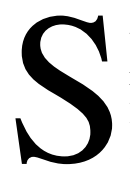

urgical treatment of Stanford type A aortic dissection remains a difficult challenge. Among of the first problems that the surgeon faces are the site and the type of arterial cannulation, which are of paramount importance in having a good arterial flow, ensuring a good body perfusion without damaging organs, not extending the dissection, speeding up surgical correction, and ensuring antegrade post-correction flow.

We developed a modified right-angled cannula that allows cannulation of the innominate artery (IA) and uniform perfusion throughout the procedure.

\section{Methods}

A preoperative computed axial tomography scan clearly identifies the extent of the dissection and the vessels involved. The right radial artery is cannulated for arterial pressure recording. A right groin incision is performed, and the femoral vessels are exposed but not cannulated. Median sternotomy is performed, and the IA is isolated. After systemic heparinization, the IA is cannulated with our modified cannula. We use a $20 \mathrm{~F}$ to $22 \mathrm{~F}$ cannula with a supplemental hole in the elbow. The width of this hole is similar to the distal cannula's hole (Figure 1). This arterial cannula is connected to the arterial line of the extracorporeal circuit. The venous return to the extracorporeal circuit is ensured by a single venous cannula in the right atrium.

Cardiopulmonary bypass is started with the arterial inflow ensured by the cannula in the IA.

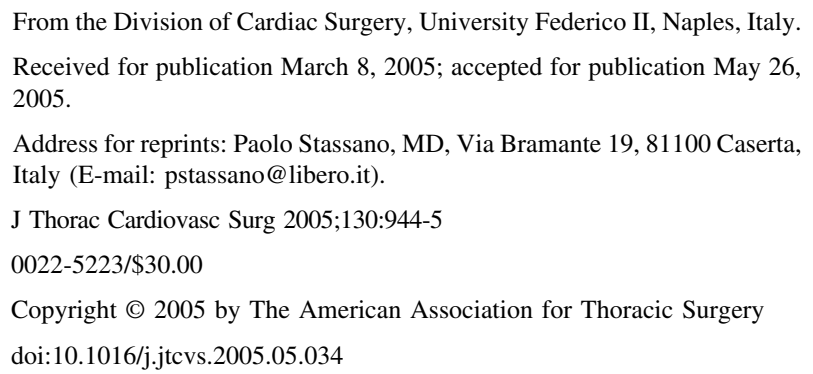

The aorta is clamped, and a longitudinal incision is made in the ascending aorta. The tear is usually seen, frequently extending into the aortic arch. Myocardial protection is achieved with intermittent blood cardioplegia delivered directly into the coronary arteries. The diseased aorta is excised, and moderate patient cooling is started. Meanwhile, the proximal aortic stump is repaired with BioGlue (CryoLife, Kennesaw, Ga), the aortic valve is resuspended, and a graft is sutured to the proximal aortic stump. At $24^{\circ} \mathrm{C}$ esophageal temperature, the IA is clamped proximally to the cannulation site (Figure 2). Cerebral perfusion through the cannula in the IA is continued at $10 \mathrm{~mL} \cdot \mathrm{kg} \cdot \min$ and adjusted to maintain a mixed venous oxygen saturation of $85 \%$ to $95 \%$, while body perfusion is momentarily stopped. The left carotid and left subclavian arteries are also clamped or occluded. Then the aorta is declamped, resection and repair of the distal ascending aorta and/or hemiarch are completed, and distal anastomosis of the tube graft is completed. With the patient in the Trendelenburg position, the flow through the IA cannula is increased gradually as the clamps on the brachiocephalic vessels are released and body perfusion is restarted exclusively through this cannula. The patient is rewarmed to $37^{\circ} \mathrm{C}$ esophageal temperature, and surgery is completed.

\section{Comment}

In type A aortic dissection many arterial cannulation sites have been proposed with the aim of providing a good and homogeneous perfusion and safe central nervous system protection.

The IA is usually spared from the dissecting lesion, easily prepared, and of sufficient caliber to allow antegrade and retrograde flow. ${ }^{1}$ The perfusion achieved with its cannulation is good and allows an excellent correction of the distal portion of the ascending aorta and/or the arch, without the cumbersome presence of the clamp. The surgical procedure can be accomplished without undue haste, because antegrade cerebral perfusion is never interrupted and the central nervous system protection is provided with a blood flow of $10 \mathrm{~mL} \cdot \mathrm{kg} \cdot \mathrm{min}$. Furthermore, deep hypothermia is not used, thus avoiding its potential hazards.

Concerns may be raised about the adequacy of cerebral perfusion, which relies on unilateral cerebral perfusion, but extensive anastomoses exist between the vertebral and the ca- 


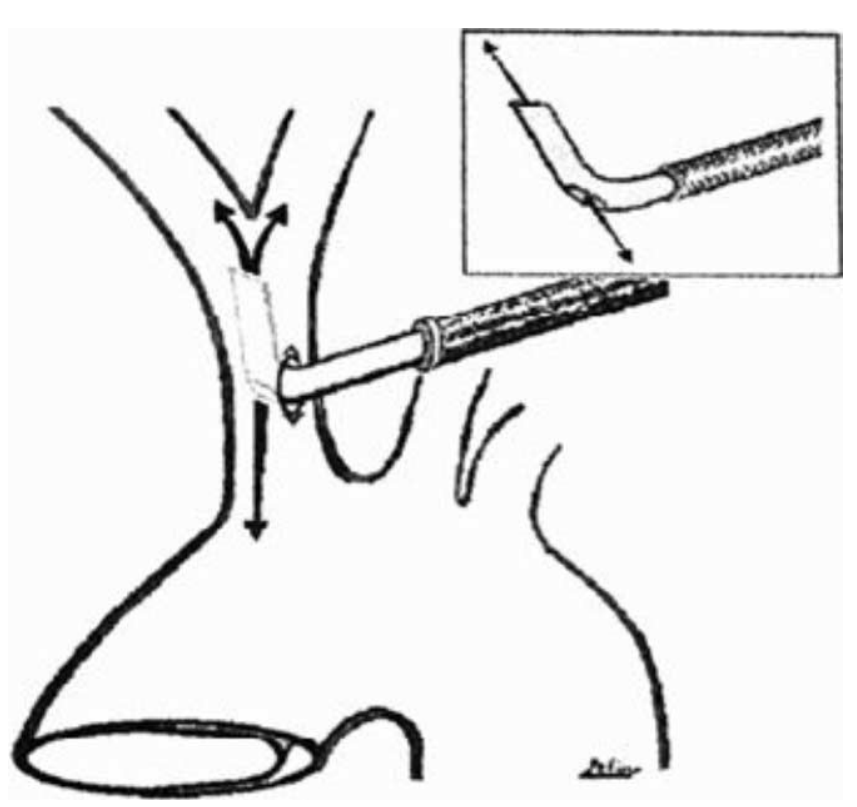

Figure 1. The IA is cannulated with the modified right-angled cannula.

rotid arteries. Frist and colleagues ${ }^{2}$ used direct perfusion of only the IA or left carotid artery with moderate hypothermia and reported no temporary or permanent deficits in their patients. Two additional studies using perfusion of the IA proved the safety of this technique..$^{3,4}$

With the IA proximally clamped, the modification in the arterial cannula allows cerebral perfusion while the systemic circulation is momentarily stopped and, with the IA declamped, antegrade systemic perfusion before and after body circulatory arrest. We have used this technique in 5 patients with type A aortic dissection, and there were no complications related to the use of this cannula or to the cannulation site. Also there were no incidences of malperfusion or cerebral complications after surgery.

In brief this cannula allows antegrade cerebral and systemic flow, does not require deep hypothermia, does not require graft

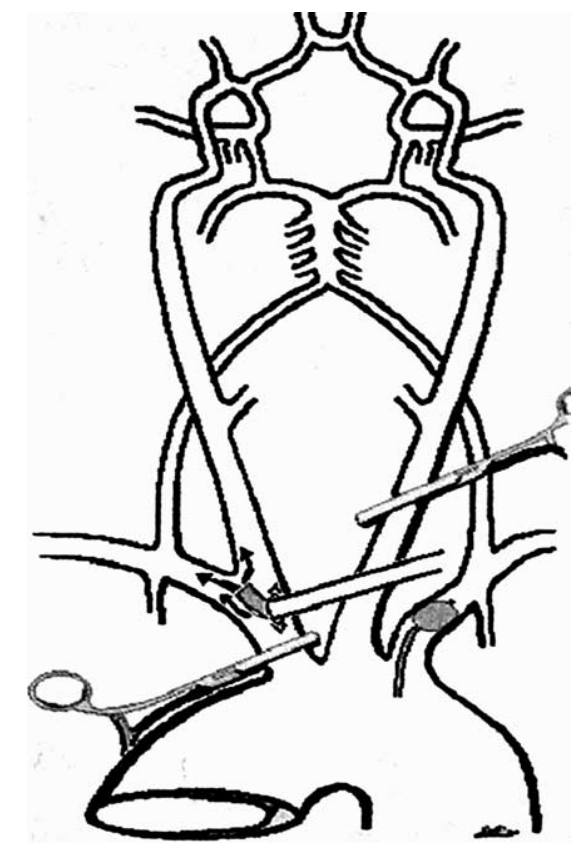

Figure 2. The IA is clamped, as well as the left carotid artery, and perfusion to central nervous system is ensured.

cannulation, ensures satisfactory brain protection, minimizes coagulation problems, and provides calm surgical correction.

\section{References}

1. Banbury MK, Cosgrove DM. Arterial cannulation of the innominate artery. Ann Thorac Surg. 2000;69:957-9.

2. Frist WH, Baldwin JC, Starnes VA, Stinson EB, Oyer PE, Miller DC, et al. A reconsideration of cerebral perfusion in aortic arch replacement. Ann Thorac Surg. 1986;42:273-81.

3. Aoyagi S, Akashi H, Kubota Y, Momosaki M, Suzuki S, Oryoji A, et al. Partial brachiocephalic perfusion in aortic arch replacement. Surg Today. 1993;23:331-7.

4. Wozniak G, Dapper F, Schindler E, Akinturk H, Zickmann B, Gehron $\mathrm{J}$, et al. An assessment of selective cerebral perfusion via the innominate artery in aortic arch replacement. Thorac Cardiovasc Surg. 1998;46: $7-11$. 